\title{
The Role of Cloud Computing in the Development of Information Systems for SMEs
}

\author{
Carlos Rompante Cunha , Elisabete Paulo Morais, João Paulo Sousa and João \\ Pedro Gomes
}

Polytechnic Institute of Bragança, EsACT, Mirandela, Portugal

Correspondence should be addressed to: Carlos Rompante Cunha;carlos.rompante@gmail.com

Received date: 7 October 2015; Accepted date: 4 April 2016; Published date: 15 February 2017

Academic Editor: Pedro Maia Malta

Copyright (C) 2017. Carlos Rompante Cunha, Elisabete Paulo Morais, João Paulo Sousa and João Pedro Gomes . Distributed under Creative Commons CC-BY 4.0

\begin{abstract}
This paper presents a review of the main characteristics of cloud computing, where they are exposed, their main components and ways of use. In addition to the technological review that is done, is also carried out. To understand how cloud computing can lead to a powerful ally of SMEs in the context of organizational competitiveness in a world where the role of information systems for a long time proved decisive, it is a reflection that the SMEs, whose core business is not technology, need to be carried out.
\end{abstract}

\section{Introduction}

Cloud Computing (CC) has been in recent years an excellent platform for the development of computational solutions for multiple fields of knowledge. This new computational approach has introduced a large number of advantages but also entails a number of challenges. The CC was a response to the current scenario where tasks such as sharing, obtaining and handling massive amounts of information are a reality. However, this scenario proves to be extremely demanding in terms of computing resources that have to exist for it is demonstrated feasible to perform these tasks with acceptable performance.
The CC has, with regard to computing resources, a vision of unlimited resources. Not because they in fact are, but by a transparently scaling approach. That is, the ability to grow cloud resources (by adding resources to a distributed cluster) shows become invisible to the end user. This just sets and engages resources, scaling this hiring according to their temporal needs without resource scarcity concerns. In fact, this is not the concern of the CC customer, but of the provider of the resources.

This way of looking at computing presents to customers (typically organizations) an important advantage. It ceases to be a concern installation, maintenance and processing infrastructure management.

Cite this Article as: Carlos Rompante Cunha, Elisabete Paulo Morais, João Paulo Sousa and João Pedro Gomes (2017)," The Role of Cloud Computing in the Development of Information Systems for SMEs", Journal of Cloud Computing, Vol. 2017 (2017), Article ID 736545, DOI:10.5171/2017.736545 
One begins to deal with a vision of computing resources, be they infrastructure, services or development platforms; as with the simple access to the Internet - it hires, binds, it is available and someone (the supplier) is responsible for ensuring the quality of service.

The CC enables organizations to focus their attention on their business, and provides them with a scalability scenario, both in terms of services and infrastructure, which become "unlimited". The cost, whose detailed analysis will not be the target of this paper, is presented as globally advantageous.

The CC came to break with the traditional view that organizations practiced, where the infrastructures are no longer proprietary organizations (something that happened for decades) and start to be seen as resources owned, managed and made available by third parties. It goes on to look at them as a sub, which takes the form of services (Carr, 2009).

This new view is reverse to what existed previously, where the ownership, management and maintenance of infrastructure resources were property of each organization. However, even if they break radically with the hitherto existing view, actually only applied the concept that organizations should focus on their core business. In this sense organizations whose business purpose is not the ICT spend in its structure, leaving others to manage their workflow, starting to focus their energies on the organization's business.

For these reasons, there are numerous challenges that the CC presents. Firstly it is a change of organizational culture regarding the way it manages the technological component. Security, sense of ownership, legal issues, among many others, are revealed as new challenges for organizations.

\section{Background}

Before delving into the concept of $\mathrm{CC}$, it becomes paramount to define it.

Cearley (2009) defines CC as a model where technological capabilities are scalable and elastic, and they are provided as a service to end-users over the Internet. The National Institute of Standards and Technology (NIST) defines the CC as a model which allows access conveniently and on-demand mode, a set of configurable computing resources (e.g. networks, servers, storage, applications and services) that can be quickly acquired and used with a reduced management effort or interaction with the service provider (Nist, 2012). Armbrust (2010) states that the CC is a set of active network services, providing scalability, quality of service, an inexpensive computing infrastructure and can be accessed in a simple and pervasive way. Second Vaquero (2008) CC can be seen as a major repository of virtualized services such as hardware and software development platform; which can be easily accessed; and that can be dynamically configured to better fit the different processing loads, and where the cost model is based on payment by the set of resources that are in fact used. To Buyya (2008), the CC is a distributed and parallel system consisting of a collection of virtualized and interconnected computers that are managed dynamically and posing as one or more unified resources. These resources are provided under the banner of a commercial agreement between a service provider and a consumer of such services, and in the background a commercial contract of use. Figure 1 illustrates a conceptual view of the CC. 


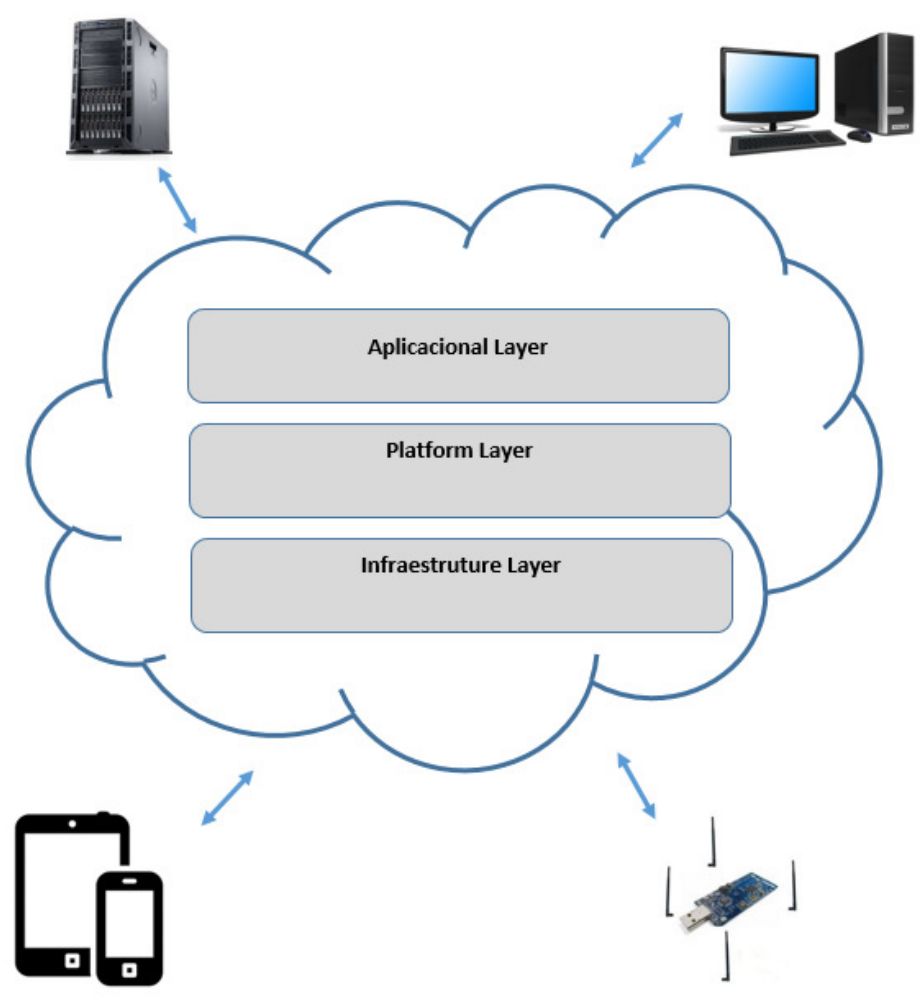

Figure 1: A conceptual view of cloud computing

\section{Cloud computing features}

The CC has a number of features that characterizes and distinguishes it from other paradigms and that are identified and briefly described below for a better understanding.

$\begin{array}{llr}\text { Resource } & \text { virtualization: } & \text { The } \\ \text { computational resources can be }\end{array}$ virtualized; there are several virtualization technologies on the market that allow you to virtualize computers, networks and storage systems. It allows to separate physical infrastructure services. This level of abstraction allows handling capabilities as services rather than physical components, making it unnecessary to its direct and physical manipulation (i.e. hardware).

Elasticity and scalability: This feature proves to be an innovation in computing and translates into the ability to deliver or remove computing resources at runtime. Associated with the elasticity of context, we have the concept of scalability which translates into the ability to increase available capacity by adding new features.

Location independence: This feature, one of the associated $\mathrm{CN}$, translates into the ability of all features that are available regardless of the user's location (and even the access device), and the existence of Internet connection which is the only necessary element for full access and manipulation of the subscribed services. Thus, the cloud is reflected in the consumer's eyes, a centralized point of access and available anywhere and in any platform.

Measuring and monitoring service: The $\mathrm{CN}$ provides monitoring of resources consumed and based the cost base by measuring these resources. Thus, it is a paradigm used at all providers of other resources used in society (e.g. electricity, gas or running water). Also, this approach is defined by the parties as a certain level 
and quality of service in order to ensure that the contracted conditions are met, and in the event of failure; penalties are applied.

Location of resources: Although the location of the resources available on the $\mathrm{CN}$ may have a dynamic dimension, it is possible to set priorities and prerogatives in relation to its location, namely the country and data center. Questions, often the legal order of each country, make this feature an important dimension in the cloud resource contracting.

\section{Service Models}

The state of the art review of $\mathrm{CC}$ has a three-tier model, as illustrated in figure 2 IaaS_PaaS_SaaS. Each layer defines a service model. The three layers are Infrastructure as a Service (IaaS), Platform as a Service (PaaS) and Software as a Service (SaaS). Next is made a brief characterization of each of these layers or service models.

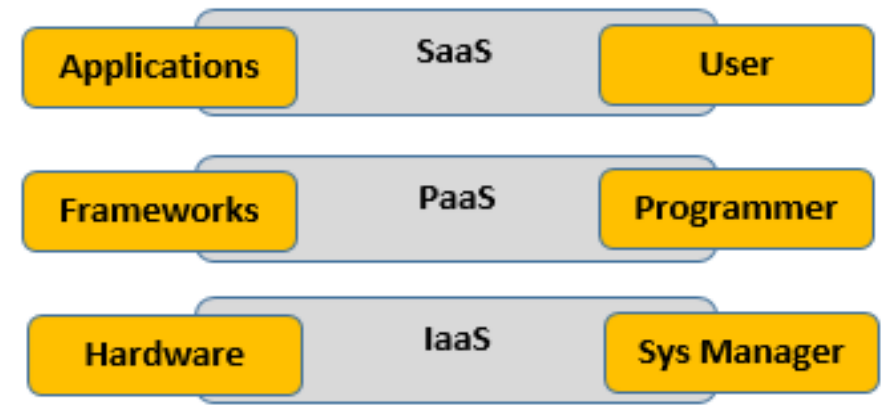

Figure 2: Services Layers of cloud computing

\section{Infrastructure as a Service (IaaS)}

This layer is the lower level of the conceptual model of the $\mathrm{CN}$. It translates the ability of the service provider must offer a computational infrastructure to a given level of processing and storage. Since this layer must necessarily be transparent to the consumer. The IaaS is typically a layer that uses virtualization engines of computing resources. This layer has the ability to dynamically increase or reduce the resources made available by virtue of the application needs; this approach is that it optimizes the maximum capacity versus capacity at a given time is not being used, i.e. the idle capacity. Some examples of IaaS are the Elastic Utility Computing Architecture Linking Your Programs To Useful Systems (Eucalyptus) (Liu, 2007) and Amazon Elastic Cloud Computing (EC2) (Robinson, 2008).

The IaaS ultimately translates into an alternative that organizations have, rather than acquiring their own computing power (which may be under- or over-sized) may contractually computing resources according to the needs of your organization, without being concerned about the need to evolve through the renovation or new equipment purchases, but by simply subscribing for greater computing capacity in the cloud. So the cloud is constituted, the consumer's eye, as one of unlimited resources platform where it is unnecessary to know how and when those resources can or should be improved.

\section{Platform as a Service (PaaS)}

It is the middle layer of the conceptual model of the CN. PaaS provides the consumer operating systems, software development platforms and programming languages, as well as storage solutions and databases. This makes it easier application development, testing, and integration services; provided that they are compatible 
with the limitations that impose these development environments, such as those available programming languages or managing systems of possible databases to use. However, it translates into a layer that greatly facilitates the development of applications. Some examples of PaaS are Aneka (Vecchiola, 2009; Vecchiola, 2012) and the Google App Engine (Ciurana, 2009; Prodan, 2012).

\section{Software as a Service (SaaS)}

This layer is the outermost layer of the CN. The SaaS represents a set of applications that run in a cloud environment. These applications are accessible through the use of a browser. This access can be done from anywhere and any device, provided there is an Internet connection available. SaaS does not require the purchase of software licenses, which reduces costs; apart to maximizing the use of ubiquitous applications. Some examples of SaaS are Google Docs, Facebook, or Microsoft SharePoint.

\section{Deployment Models}

The implementation of the $\mathrm{CN}$ or more specifically the creation of clouds can come under different models. The state of the art $\mathrm{CN}$ refers to private clouds, public clouds, community clouds, and hybrid clouds. Each has its own characteristics that are then characterized and briefly illustrated in figure 3.

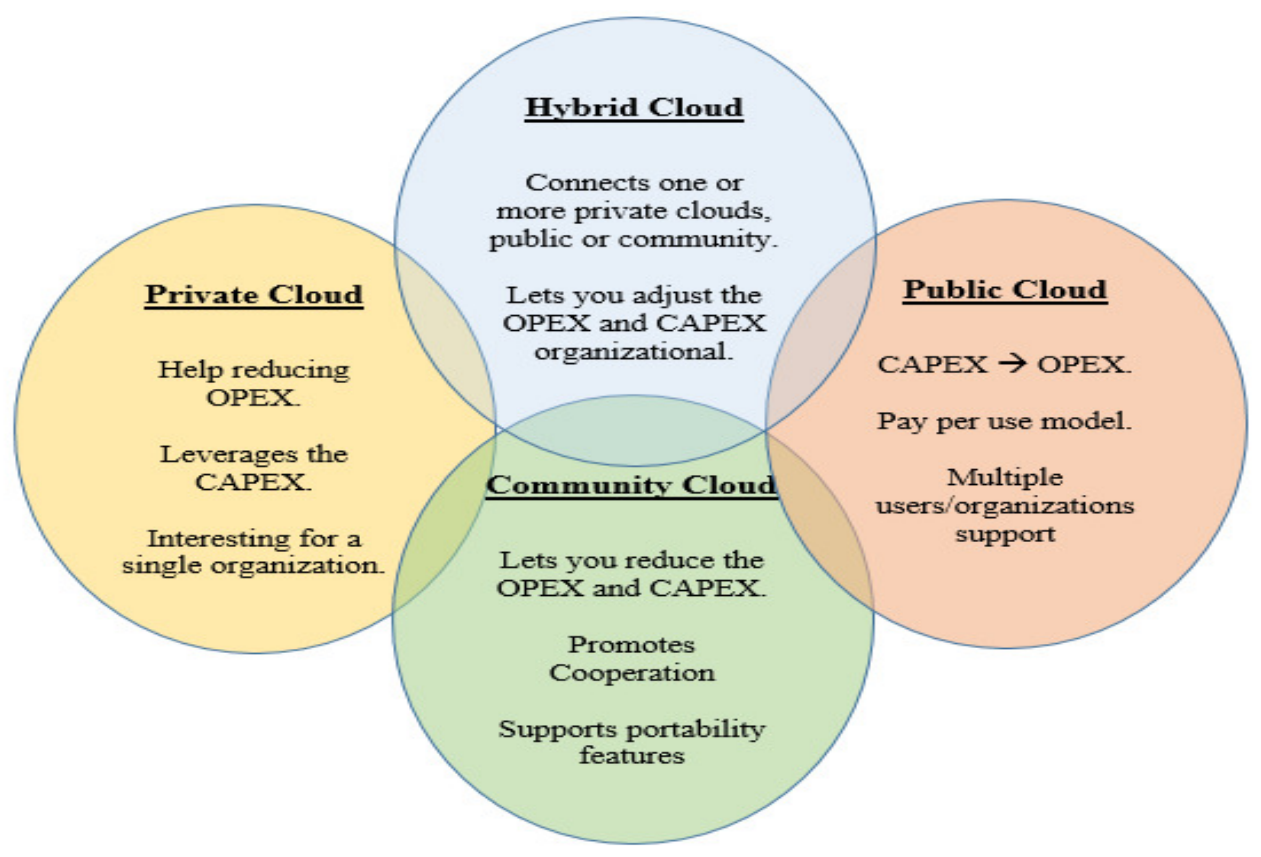

Figure 3: Cloud computing implementation models

\section{Private Cloud}

In this model the cloud infrastructure is proprietary and is used exclusively by the organization that owns it. The issues related to access control and its restrictions featuring this style. Associated with this model is normally the high cost of creating and maintaining these clouds over the use of a public cloud. However, it should be noted the largest and most detailed control is over the resources that form the cloud. In this model the organization has absolute control over all configuration options of computing resources and existing service. 


\section{Public Cloud}

In this model, the cloud appears as a shared resource space for a set of organizations that normally have common interests. These common interests will tend to converge to harmonize requirements and security policies, and aspects of flexibility of use.

Typically, in this model, one of the organizations assumes the role of cloud management. This scenario may be interesting in scenarios of cooperation networks, it is noteworthy that combined with common interests, a cooperation network can share the costs of creating and maintaining a cloud and combine the advantages inherent in greater control and configuration of cloud resources in the face of public clouds and / or contracted to third parties.

\section{Community Cloud}

In this model, the cloud appears as a shared resource space for a set of organizations that normally have common interests. These common interests will tend to converge to harmonize requirements and security policies, and aspects of flexibility of use.

Typically, in this model, one of the organizations assumes the role of cloud management. This scenario may be interesting in scenarios of cooperation networks, it is noteworthy that combined with common interests, a cooperation network can share the costs of creating and maintaining a cloud and combine the advantages inherent in greater control and configuration of cloud resources in the face of public clouds and / or contracted to third parties.

\section{Hybrid Cloud}

This model is, of course, difficult to implement since it is materialized in practice, the configuration of two clouds. One dedicated to critical processes of the organization and another dedicated to secondary processes. Thus, it can create a private domain with high control security and internal nature; and another domain cloud over public nature, or at least less subject to tight control and more oriented to withstand very demanding process environments. It is a hybrid solution difficult to achieve, especially because is difficult to cohabit and interconnect these two clouds.

\section{Discussion and Final Remarks}

Most SMEs are not technological companies as such are more focused on their core business and less available to address the technological aspects of support information processing and computer services. However, it is unanimously accepted by any modern organization that information is a valuable enabler of competitiveness and success. But how can organizations that do not have resources necessary to acquire the latest technology, nor develop a structure, be able to manage and evolve in a scalable way the technological component? We believe that the $\mathrm{CC}$ is responding to this challenge. The CC allows SMEs to transfer the technology infrastructure to a third party (the supplier of a technological cloud) fitting to this part of the whole management, scalability, and development of infrastructure. For an SME it is a vision where technology becomes a piece that will "never" become obsolete. That someone will have to manage and account for its full operability. Where this someone has to keep resources always adjusted to the real organization's needs. The view, under a pay per use basis, that what once was an IT department becomes a contracted service to third parties. Well, necessarily this vision can never be exactly like this, as they are always necessary internal resources of the organization itself that will use the capabilities of the cloud. However, many of the problems will be set aside and that's very positive for most SMEs who have neither resources nor profiles for themselves to face the technological challenges surrounding organizations, whatever their business activity.

However, legal issues about where the computing resources are physically located primarily in the public cloud, raising trust issues regarding the deposit of sensitive 
information from companies. In fact, the laws that govern the country where the information is headquartered that decide the reliability of the information. Often these laws can be very different from the laws of the home organization, which uses the cloud.

\section{References}

1. Armbrust, M., Fox, A., Griffith, R., Joseph, A. D., Katz, R., Konwinski, A., Lee, G., Patterson, D., Rabkin, A., Stoica, I., e Zaharia, M. (2010). A view of Cloud computing. Commun. ACM, 53(4):50-58.

2. Buyya, R., Yeo, C. S., e Venugopal, S. (2008). Market-oriented cloud computing: Vision, hype, and reality for delivering it services as computing utilities. In Proceedings of the 2008 10th IEEE International Conference on High Performance Computing and Communications, HPCC '08, 5-13, Washington, DC, USA. IEEE Computer Society.

3. Carr, N. (2009). The Big Switch: Rewiring the World, from Edison to Google. W.W. Norton \& Company.

4. D. Cearley et al (2009). Hype cycle for applications development gartner group reporter number G00147982, Gartner.

5. Ciurana, E. (2009). Developing with Google App Engine. Apress, Berkely, CA, USA, 1st Edition.
6. Liu, S., Liang, Y., e Brooks, M. (2007). Eucalyptus: A web service-enabled einfrastructure. In Proceedings of the 2007 Conference of the Center for Advanced Studies on Collaborative Research, CASCON '07,1-11, Riverton, NJ, USA. IBM Corp.

7. NIST (2012). The NIST Definition of Cloud Computing, Gartner.

8. Prodan, R., Sperk, M., e Ostermann, S. (2012). Evaluating high-performance computing on google app engine. IEEE Softw., 29(2):52-58.

9. Robinson, D. (2008). Amazon Web Services Made Simple: Learn How Amazon EC2, S3, SimpleDB and SQS Web Services Enables You to Reach Business Goals Faster. Emereo Pty Ltd, London, UK.

10.Vaquero, L. M., Rodero-Merino, L., Caceres, J., e Lindner, M. (2008). A break in the clouds: Towards a cloud definition. SIGCOMM Comput. Commun. Rev., 39(1):50-55.

11.Vecchiola, A. C., Chu, A. X., e Buyya, A. R. (2009). Aneka: A Software Platform for .NET-based Cloud Computing.

12.Vecchiola, C., Calheiros, R. N., Karunamoorthy, D., e Buyya, R. (2012). Deadlinedriven provisioning of resources for scientific applications in hybrid clouds with aneka. Future Generation Comp. Syst., 28(1):58-65. 